\title{
Plataforma de investigación para el confinamiento virtual de bovinos
}

\author{
Research platform for the virtual confinement of bovines
}

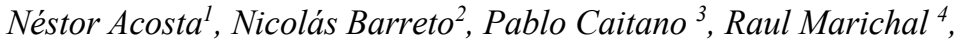 \\ Martín Pedemonte ${ }^{5}$, Julián Oreggioni ${ }^{6}$
}

Recibido: Febrero 2021

Aceptado: Marzo 2021

Resumen.- Existen varios antecedentes de alambrados virtuales que utilizan diversas técnicas para mantener confinado al ganado bovino dentro de un perímetro configurado de forma remota. Estas técnicas comparten un patrón común que consiste en colocar un dispositivo electrónico en el animal capaz de aplicar estímulos cuando éste se acerca a los límites pre-establecidos. El uso de descargas eléctricas como método de estimulación es ampliamente utilizado. En este trabajo se propone una solución compatible con el bienestar animal, que evita las descargas eléctricas, basada solamente en estímulos sonoros y táctiles (mediante un motor vibrador). Para ello, se desarrolló un sistema que comprende un dispositivo electrónico que se coloca en el cuello del animal, y es capaz de determinar su posición, estimularlo y enviar información en forma inalámbrica; un servidor central que es capaz de recibir, procesar y almacenar esa información; y una interfaz gráfica, a través de la cual se puede visualizar la posición del animal y configurar distintos parámetros para evaluar diversas metodologías de confinamiento virtual. Las pruebas realizadas en animales sugieren que los estímulos propuestos no son inocuos, por lo que se estima, podrían lograr su cometido luego de un periodo de aprendizaje de los animales. La investigación sobre la eficacia de las metodologías de confinamiento de la plataforma desarrollada será realizada en una próxima etapa por un equipo interdisciplinario.

Palabras clave: Alambrado virtual, confinamiento animal, seguimiento de animales, bienestar animal

Summary.- Prior work in virtual fences has proposed different schemes to keep cattle confined within a remotely configured perimeter. These techniques share a common pattern that consists of placing an electronic device in the animal capable of applying stimuli when it approaches the preestablished limits. The use of electric shocks as a method of stimulation is widely used. This work

\footnotetext{
${ }^{1}$ Instituto de Ingeniería Eléctrica, Facultad de Ingeniería, Universidad de la República, ngacosta16@gmail.com, ORCID iD: https://orcid.org/0000-0002-7400-8131

2 Instituto de Ingeniería Eléctrica, Facultad de Ingeniería, Universidad de la República, na.barretovalentin@gmail.com , ORCID iD: https://orcid.org/0000-0001-8891-6731

3 Instituto de Ingeniería Eléctrica, Facultad de Ingeniería, Universidad de la República, pablocaitano5@gmail.com, ORCID iD: https://orcid.org/0000-0002-0346-5976

4 Instituto de Computación, Facultad de Ingeniería, Universidad de la República, rmarichal@fing.edu.uy, ORCID iD: https://orcid.org/0000-0003-4974-0803

5 Instituto de Computación, Facultad de Ingeniería, Universidad de la República, mpedemon@fing.edu.uy, ORCID iD: https://orcid.org/0000-0002-8348-0921

6 Instituto de Ingeniería Eléctrica, Facultad de Ingeniería, Universidad de la República, juliano@fing.edu.uy, ORCID iD: https://orcid.org/0000-0003-0164-6877
}

Memoria Investigaciones en Ingeniería, núm. 20 (2021). pp 2-15

https://doi.org/10.36561/ING.20.2

ISSN 2301-1092 • ISSN (en línea) 2301-1106 
proposes a solution compatible with animal welfare, which avoids electric shocks, based only on sound and tactile stimuli (using a vibrating motor). For this, a system was developed consisting of an electronic device that is placed on the animal's neck, and has the capacity to stimulate and send information wirelessly; a central server that is able to receive, process and store that information; and a graphical user interface, where the animal's position can be visualized and several parameters can be configured to evaluate different virtual confinement techniques. The tests performed on animals suggest that the stimuli used is aversive, so it is estimated that they could achieve their goal after a period of training. The research on the effectiveness of the proposed confinement techniques using our platform should be carried out in a next stage by an interdisciplinary team.

Keywords: animal confinement, cattle tracking, animal welfare 
Introducción.- Los alambrados virtuales (AV) han sido objeto de estudio desde hace varias décadas. Su utilización para el confinamiento animal, en sustitución de las barreras físicas usadas hoy en día, puede implicar grandes beneficios para la industria ganadera [1]. Los AV se basan en la colocación de dispositivos electrónicos con GPS en los animales, que son capaces de aplicar estímulos cuando algún animal se acerca a los límites pre-establecidos. En particular, el uso de descargas eléctricas es ampliamente utilizado como método de estimulación. Este tipo de sistemas típicamente incorpora telemetría, por lo que permite monitorear a los animales y estudiar su comportamiento [2]-[3], así como también lograr la reubicación de los animales, permitiendo la aplicación de técnicas de pastoreo de precisión [4]-[7]. Asimismo, pueden proporcionar la ubicación del animal en tiempo real de manera continua, lo que permite ayudar a reducir los casos de extravío y abigeato, y a la detección temprana de enfermedades (por ejemplo, mediante el estudio patrones de movimiento). A su vez, la implantación de estos sistemas puede provocar una reducción importante en los costos de mantenimiento con respecto al alambrado tradicional físico. Incluso en casos de emergencia, como inundaciones o incendios, puede contribuir a evitar pérdidas significativas, dado que se evitan obstáculos que retengan a los animales en lugares con potencial peligro.

Si bien recientemente se han obtenido resultados alentadores que validan la eficacia de las técnicas de AV [8]-[9], la utilización de estímulos como las descargas eléctricas generan controversia e incluso se han llegado a prohibir en algunos países [10]. En este contexto, el estudio de técnicas compatibles con el bienestar animal puede brindar nuevas oportunidades para el desarrollo y posterior aplicación de esta tecnología.

El objetivo de este trabajo consiste en generar una plataforma de investigación que permita evaluar diferentes técnicas de confinamiento virtual utilizando estímulos compatibles con el bienestar animal. En este sentido, se decidió comenzar usando estímulos sonoros y táctiles (vibracionales).

Este trabajo es una versión actualizada y traducida al español de un trabajo previo de los autores [11] asociado a una tesis de grado [12] de Ingeniería Eléctrica de la Universidad de la República. El proyecto actualmente está siendo comercializado por la empresa Catel [13] y se enmarca dentro de una línea de investigación activa entre grupos de las Facultades de Ingeniería y de Veterinaria de la Universidad de la República.

1. Solución propuesta.- La Fig. I muestra un esquema de la plataforma propuesta. El núcleo de nuestra plataforma de cercas virtuales es un dispositivo electrónico colocado en los animales llamado nodo sensor (NS). El NS se basa en un microcontrolador de bajo consumo de energía con los siguientes requerimientos de diseño:

- La estimulación generada por el NS debe ser compatible con el bienestar animal (por ejemplo, estímulos sonoros y táctiles/vibratorios).

- Debe poder determinar su ubicación geográfica en tiempo real con una precisión de $5 \mathrm{~m}$.

- Debe comunicarse de forma inalámbrica con un servidor central mediante un protocolo de largo alcance y bajo consumo de energía. Dado que el perímetro virtual debe ser configurable desde el servidor central, la comunicación debe ser bidireccional y tener un alcance mínimo de $5 \mathrm{~km}$.

- Se busca que el NS tenga un área menor a $225 \mathrm{~cm} 2$, una autonomía mínima de 7 días y una frecuencia de muestreo de la ubicación de al menos $1 \mathrm{~Hz}$. 


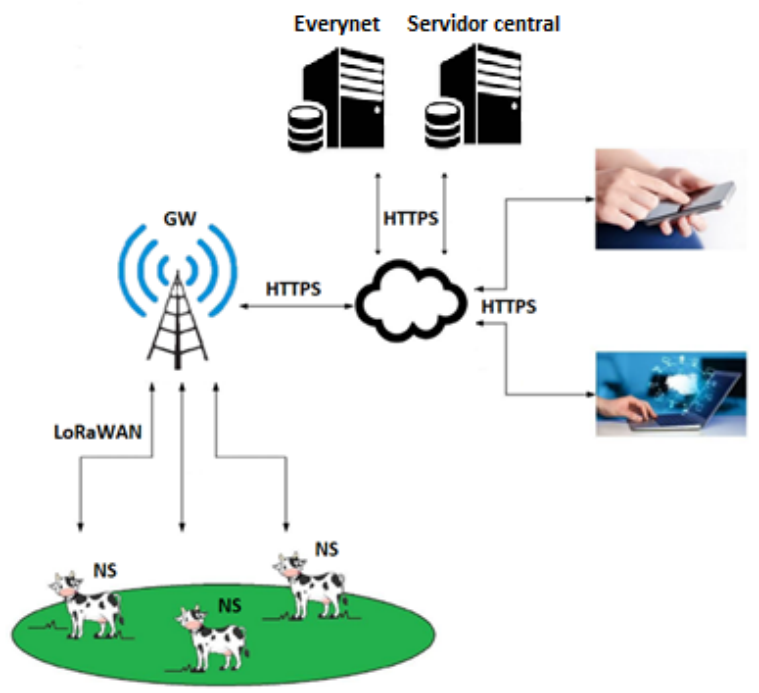

Figura I-Arquitectura del sistema propuesto.

Dados los requerimientos de consumo y alcance, se definió utilizar la tecnología LoRa para la transmisión de datos y LoRaWAN como protocolo de red. Este protocolo establece una topología de red en estrella, definiendo tres clases de dispositivos (A, B y C) para la comunicación entre múltiples nodos y un gateway ( $\mathrm{GW}$, por su sigla en inglés). Los dispositivos clase A soportan comunicación bidireccional y pueden enviar mensajes en cualquier momento, mientras que la recepción sólo podrá ocurrir luego del envío de un mensaje. Los dispositivos clase $\mathrm{B}$ y $\mathrm{C}$, son extensiones de la clase A, que ofrecen periodos de recepción más amplios, a costa de un mayor consumo. Dadas las características buscadas para el dispositivo se optó por trabajar con la clase A. Se implementó el protocolo LoRaWAN utilizando la biblioteca IBM LMiC [14], que se describe en Sección II-B. Esto requiere que el microcontrolador del NS deba tener una memoria de programa superior a $32 \mathrm{kB}$.

La arquitectura de nuestra solución contempla que el sistema se utilice simultáneamente en varios animales. Por lo tanto, la red de NS se comunica con el servidor central a través de uno o varios gateways. Los gateways se comunican con el servidor central utilizando la plataforma de Everynet. El servidor central proporciona los servicios de backend para almacenar la información en una base de datos gestionando la comunicación con los NS. También proporciona una interfaz gráfica de usuario (frontend) para visualizar los NS en un mapa y configurar los diferentes parámetros de la plataforma.

1.1. Hardware.- El sistema embebido fue diseñado usando la placa de desarrollo Moteino basada en Arduino. Esta placa incluye un microcontrolador ATmega 1284P y una radio RFM95 para comunicación. Además, se incorporó un módulo GPS, un buzzer, un motor DC (vibrador) y un sistema de gestión de energía. La Fig. II presenta el diagrama de bloques del NS.

El microcontrolador ATmega 1284P de la familia AVR de Microchip presenta un consumo de 400 $\mu \mathrm{A}$ en "Active Mode" a $1 \mathrm{MHz}$ y $0.1 \mu \mathrm{A}$ en "Power-down Mode". Funciona con un bus de datos de 8 bits, tiene $128 \mathrm{kB}$ de memoria Flash, 4 kB de EEPROM y $16 \mathrm{kB}$ de memoria SRAM. Además, incluye hasta 32 pines de entrada/salida.

Para determinar la ubicación del NS se escogió el módulo GPS NEO-M8N del fabricante u-blox. Los mismos tienen una precisión de $2.5 \mathrm{~m}$ en condiciones óptimas y una frecuencia de muestreo máxima de $10 \mathrm{~Hz}$. 
Se optó por un módulo de comunicación RFM95, basado en el chip SX1276 de Semtech, el cual utiliza LoRa. Este módulo tiene un consumo de $10 \mathrm{~mA}$ y $120 \mathrm{~mA}$ en recepción y transmisión respectivamente, y puede alcanzar sensibilidades de hasta $-148 \mathrm{dBm}$ usando un cristal de bajo costo.

El sistema embebido cuenta con dos mecanismos diferentes de estimulación, basados en sonido y vibraciones. El estímulo sonoro se implementa mediante un buzzer pasivo. El microcontrolador puede modificar la frecuencia del buzzer variando la frecuencia de una señal auxiliar (hasta 10 $\mathrm{kHz}$ ). Además, se utiliza una señal PWM para generar diferentes niveles de voltaje en su fuente de alimentación con el fin de modificar su volumen (que puede superar los $100 \mathrm{dBA}$ ). Por otro lado, el estímulo vibratorio se genera con un motor R260 de corriente continua, con una velocidad de hasta $3000 \mathrm{rpm}$ y un peso de 30 gramos.

El sistema de energía está compuesto por un panel solar rígido de 4,5 V y 0,5 W conectados en serie con dos paneles solares flexibles de $2 \mathrm{~V}$ y $0,5 \mathrm{~W}$, una batería Li-Ion 18650 de capacidad 3400 mAh y tensión nominal 3,7 V, y un cargador de batería Li-Ion lineal TP4056 con una corriente máxima de $500 \mathrm{~mA}$.

El gateway elegido fue un Everynet Network Gateway v2.0, compatible con LoRaWAN. Dicho gateway tiene un GPS integrado y se puede conectar a Internet utilizando una red de datos móviles $3 \mathrm{G}$ o Ethernet. Es alimentado por el puerto Ethernet (PoE, Power over Ethernet), y tiene una batería de respaldo integrada. El mismo cuenta con un rango nominal de más de $15 \mathrm{~km}$ con línea vista y de hasta $2 \mathrm{~km}$ en zonas urbanas densamente pobladas.

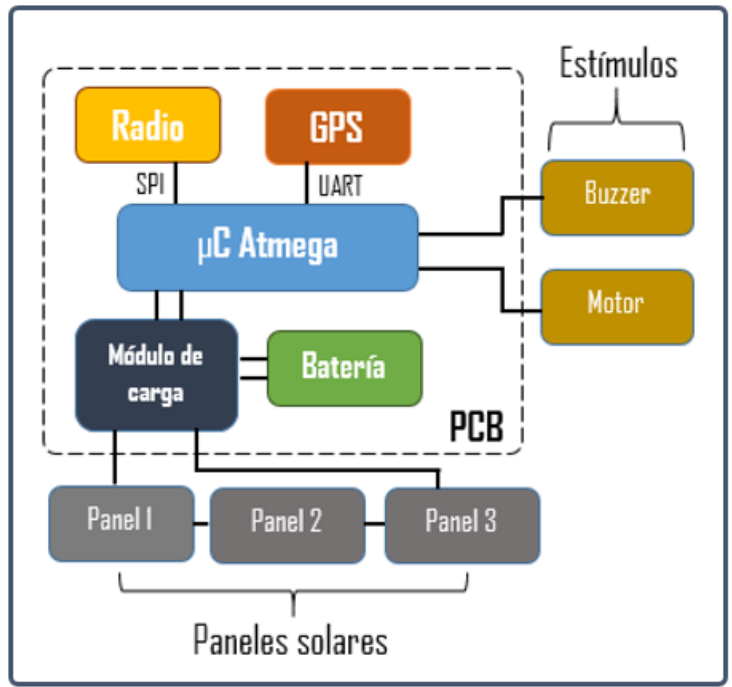

Figura II - Diagrama de bloques del SN.

1.2. Software Embebido.- El software embebido del NS fue desarrollado en $\mathrm{C}++$, usando funciones de Arduino. La implementación está fuertemente basada en la biblioteca LMiC [14]. $\mathrm{LMiC}$ es una implementación en lenguaje $\mathrm{C}$ de la especificación de la capa de control de acceso al medio del protocolo LoRa. La biblioteca utiliza un modelo de programación basada en eventos, donde el código de la aplicación es ejecutado por tareas, que son activadas por eventos y se gestionan mediante funciones de la biblioteca. LMiC incluye controladores para la radio Semtech SX1276, y también una capa de abstracción de hardware, que ayuda a utilizarlo con otros módulos de radio. En particular, se utilizó la biblioteca implementada por M. Kooijman [15] para incorporar la radio RFM95 con la biblioteca LMiC en el entorno de Arduino. 
En la Fig. III se puede observar el flujo del software embebido. En primer lugar, se adquieren la posición (latitud y longitud), y la fecha y hora. Luego se calcula la distancia hacia cada uno de los laterales del perímetro virtual y se determina si se encuentra dentro o fuera del mismo. Esto se realiza mediante el algoritmo "Ray Casting" [16]. En base a esta información el sistema decide entre dos modos de funcionamiento: Modo de Estimulación y Modo de Transmisión de Datos (bajo consumo).

Cuando el NS se encuentra lejos del perímetro virtual se entra en el Modo de Transmisión de Datos. En este modo se transmite el mensaje correspondiente, y de acuerdo al funcionamiento del protocolo LoRa clase A, se abren dos ventanas de tiempo (uno y dos segundos luego de la transmisión, respectivamente) para habilitar la recepción de mensajes desde el servidor central. En caso de no recibir datos, el microcontrolador, la radio y el GPS pasan a un estado de bajo consumo durante un tiempo configurable.

El esquema del perímetro virtual consta de dos zonas de estimulación diferentes con un ancho configurable: la zona F1, donde sólo el buzzer está funcionando, y la zona F2, donde el buzzer y el vibrador están activados (ver Fig. IV). Cuando el NS está cerca del perímetro virtual, el dispositivo entra en el Modo de Estimulación y el animal es estimulado usando uno o ambos mecanismos dependiendo de la zona en la que se encuentre. En este modo, los datos del GPS se adquieren con la máxima frecuencia y se inhabilita la comunicación para aumentar la velocidad de respuesta del dispositivo a los movimientos del animal.

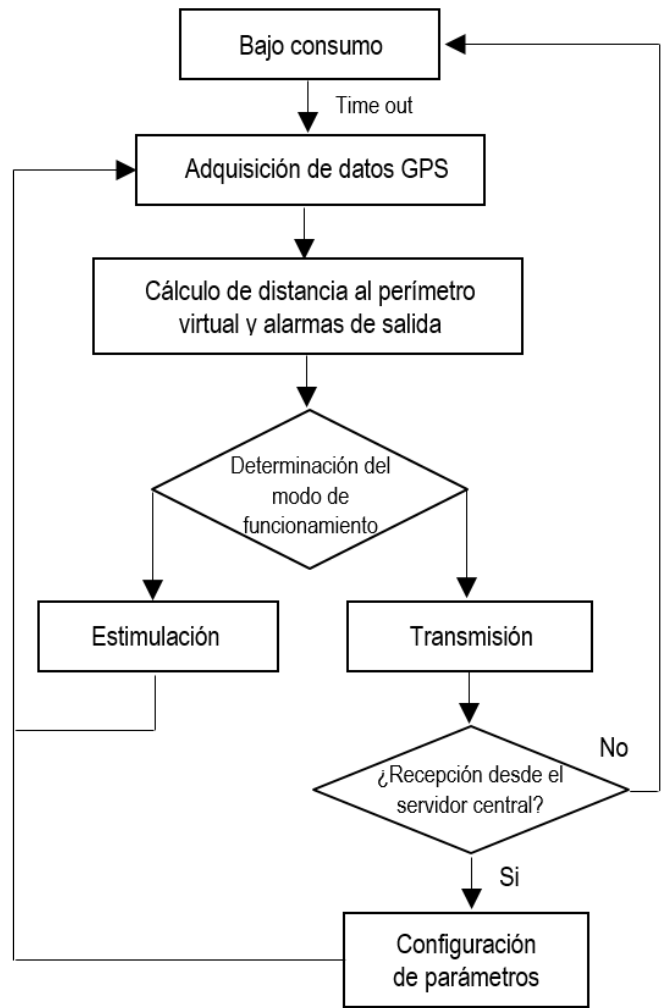

Figura III.- Diagrama de flujo simplificado del firmware del NS 


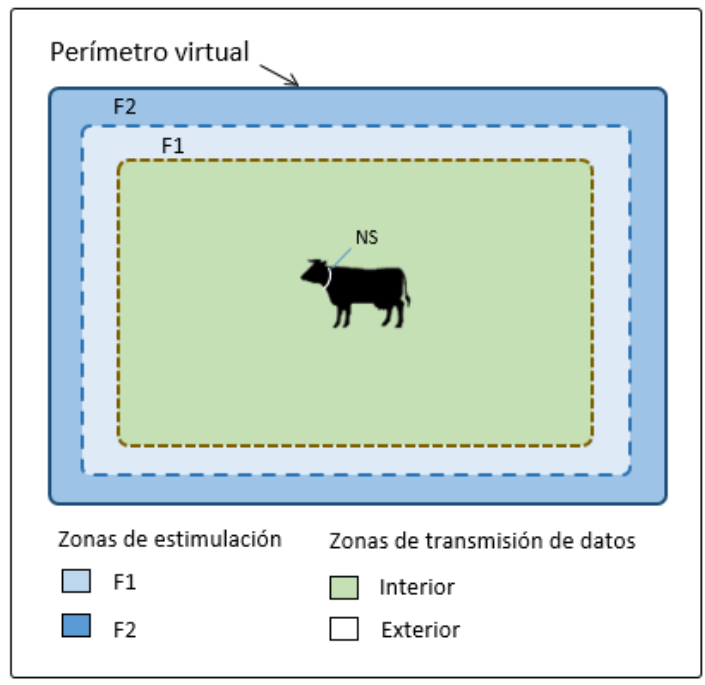

Figura IV-Zonas del Perímetro Virtual

1.3. Software.- Para simplificar la interacción entre el usuario y los dispositivos, se desarrolló una aplicación web alojada en la nube. El sistema sigue la arquitectura sugerida por EveryNet (ver Fig. 1) y está dividido en tres partes principales: frontend, backend y base de datos (ver Fig. V). Los NS envían información periódicamente al gateway, que luego se carga en la plataforma Everynet a través del protocolo HTTPS. Esta plataforma tiene una API a la que se puede acceder a través de un servicio web.

El backend consume la API de datos expuesta por Everynet utilizando una interfaz de transmisión bidireccional basada en WebSockets, diseñado para el intercambio de mensajes entre el servidor de EveryNet y nuestra aplicación. De esta forma, el backend periódicamente recibe datos de los NS (uplinks) y los almacena en la base de datos. El backend también puede enviar al servidor de EveryNet comandos de configuración para los dispositivos, recibidos previamente desde el frontend (downlink).

El frontend proporciona una interfaz de usuario para rastrear y configurar cada NS. Se comunica con el backend para obtener información de los dispositivos (ubicación, configuración, etc.) y enviar nuevas configuraciones. También consume la API de Google Maps para crear perímetros (cercas virtuales) y mostrar las ubicaciones actuales e históricas en un mapa. La interfaz de usuario se muestra en la Fig. VI. 


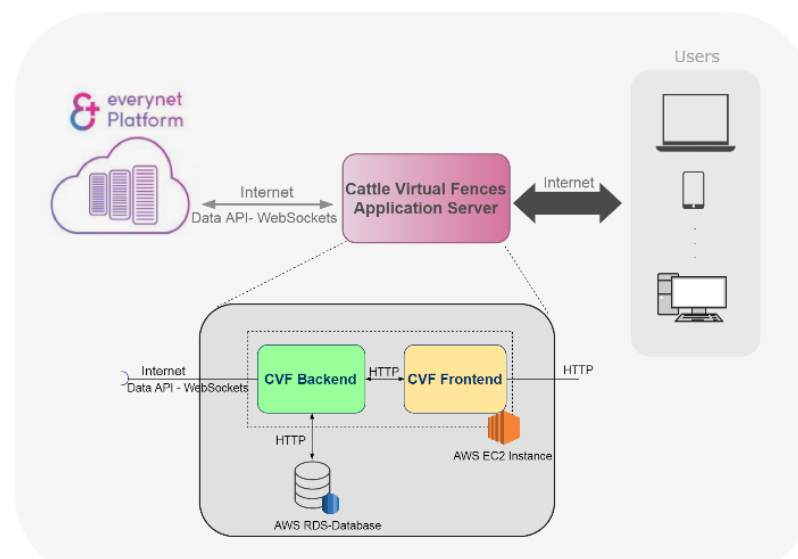

Figura V. Arquitectura del servidor de aplicaciones

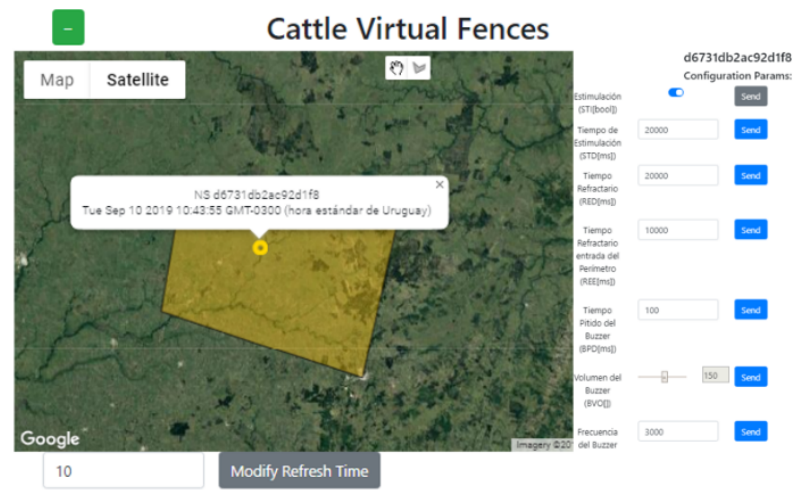

Figura VI. Ubicación de un NS y su perímetro virtual configurado en la interfaz de usuario.

Las principales funcionalidades de la aplicación son:

- Alta y baja de cercas virtuales para cada NS.

- Configuración de parámetros para cada NS: perímetro virtual, ancho de las zonas de estimulación, período de muestreo en el modo de transmisión de datos, tiempo de estimulación, frecuencia y volumen de estímulo sonoro.

- Muestra la ubicación actual de cada NS.

- Muestra la ubicación histórica de cada NS.

1.4. Fabricación.- Los NS deben sujetarse al cuerpo del animal para ser capaces de informar su ubicación continuamente. Se tuvo especial cuidado en algunos aspectos del diseño del dispositivo como ser el tamaño, peso y método de sujeción, para no causar daño a los animales. Por esta razón, se fabricó un collar con cinta mochila y hebillas de plástico, que se puede ajustar usando un velcro, como se puede ver en la Fig. VII. El rango de ajuste es de 70 a $90 \mathrm{~cm}$. La cinta mochila es lo suficientemente flexible para sujetarse al cuello del animal y también se puede perforar fácilmente, lo que simplificó su costura.

En la etapa de diseño, se decidió separar el motor vibrador del bloque principal (ver Fig. 7). Debido a esto, fue necesario diseñar dos contenedores diferentes, uno para el bloque principal (que incluye el microcontrolador, GPS, radio LoRa, buzzer, baterías y panel solar) y otro para el motor. También 
incluimos un mecanismo de contrapeso en el collar para asegurar que los paneles solares estén siempre orientados hacia el sol.

El diseño de los encapsulados se realizó con el software Tinkercad de Autocad. Para su impresión se optó por trabajar con PLA (ácido poliláctico) por ser un material resistente, económico y de naturaleza biodegradable. Las piezas impresas fueron pulidas para mejorar su acabado y permitir una mejor colocación de los componentes en su interior. Se rellenaron los orificios de entrada de los cables con silicona para evitar el ingreso de agua.

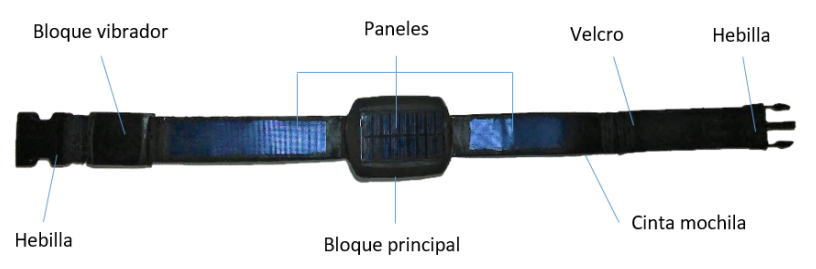

Figura VII. Prototipo final del NS. Se indican en la figura el bloque principal, el bloque vibrador, los paneles solares, hebillas y velcro de sujeción.

2. Resultados.- En esta sección se presentan y analizan los resultados experimentales del prototipo del sistema de cercas virtuales para ganado bovino diseñado en este trabajo. En particular, se analizan varias características importantes de la plataforma, incluyendo el consumo de corriente, la autonomía y su rango de comunicación.

2.1. Consumo.- El consumo individual de cada componente se obtuvo con el medidor de corriente Otii Standard de Qoitech. En la Fig. VIII se presenta el perfil de consumo en el Modo de Transmisión de Datos (bajo consumo). En base a estas medidas experimentales se calcularon los ciclos de trabajo de cada componente (que es el \% del tiempo que el componente está activo, el resto del tiempo está apagado o en un estado de bajo consumo de energía), para evaluar el tiempo que cada uno de ellos está operativo (ver Tabla I). Finalmente se determinó el consumo promedio del dispositivo bajo el supuesto de que estaría un $80 \%$ del tiempo funcionando en el modo de transmisión de datos (bajo consumo), llegando a un valor de consumo promedio de $28.1 \mathrm{~mA}$.

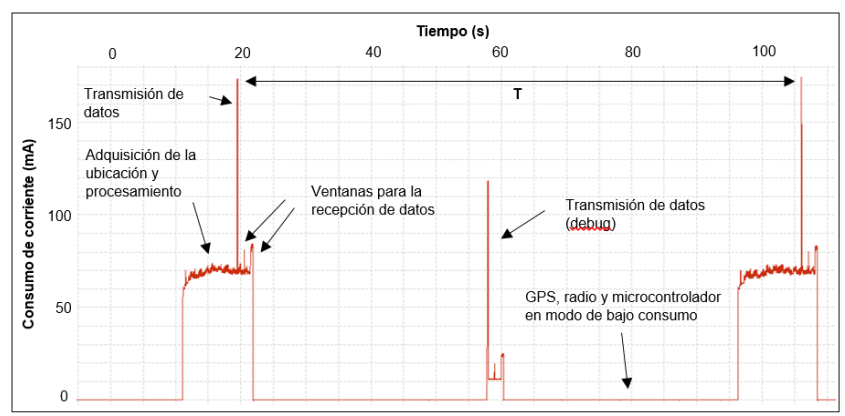

Figura VIII. Perfil de consumo del NS, en el modo de bajo consumo. Entre transmisiones de datos se ve una transmisión de datos para control y debugueo. 


\begin{tabular}{lcc}
\hline Componente & Consumo & Ciclo de trabajo \\
\hline Atmega 1284p & $11 \mathrm{~mA}$ & $36 \%$ \\
Radio RFM95 stand-by & $2 \mathrm{~mA}$ & $36 \%$ \\
Radio RFM95 TX & $120 \mathrm{~mA}$ & $0.09 \%$ \\
Radio RFM95 RX & $10 \mathrm{~mA}$ & $0.27 \%$ \\
GPS NEO M8N & $57 \mathrm{~mA}$ & $32.8 \%$ \\
Buzzer & $47 \mathrm{~mA}$ & $2.5 \%$ \\
Motor & $540 \mathrm{~mA}$ & $0.625 \%$ \\
\hline
\end{tabular}

Tabla I. Consumo promedio de componentes.

2.2. Autonomía.- Se considerará ahora la autonomía del dispositivo. Con un consumo promedio de $28,1 \mathrm{~mA}$, se requieren $674,4 \mathrm{mAh}$ diarios. Por tanto, utilizando una batería de 3,4 Ah, la autonomía del equipo es de 5,1 días en ausencia total de luz solar.

La corriente entregada por los paneles solares se midió con un Multímetro DT9208L. Se obtuvo un valor promedio de $100 \mathrm{~mA}$ con valores pico de $120 \mathrm{~mA}$, con un voltaje constante de 4,2 V, proporcionado por el módulo de carga TP4056. Se concluye que los paneles seleccionados son capaces de cosechar $100 \mathrm{mAh}$ por hora de luz solar. Como consecuencia, se necesitan aproximadamente 7 horas de sol diarios para cubrir los 674,4 $\mathrm{mAh}$ requeridos. Esto es factible de alcanzar casi todo el año en Uruguay, salvo en invierno. Si se considera un peor caso (invierno), donde se obtienen en promedio 3 horas de sol al día, la autonomía llegaría a los 10 días.

2.3. Alcance.- Se realizaron dos pruebas diferentes para analizar el rango de comunicación entre el NS y el gateway. Primero, se llevó a cabo un experimento con línea vista en un entorno urbano. Los resultados experimentales muestran que la comunicación tiene un rango de al menos $9 \mathrm{~km}$. En el segundo experimento, el gateway fue instalado en el techo de un galpón de granja. En este caso, hubo obstáculos (principalmente grupos de árboles y construcciones) entre el NS y el gateway, y la distancia máxima de comunicación alcanzada fue de 1,6 km. En ambos casos se utilizó un spreading factor de 7. Los resultados se presentan en la Tabla II.

\begin{tabular}{lrrl}
\hline Área & Altura GW & Alcance & Medio \\
\hline Rural & $4 \mathrm{~m}$ & $1.6 \mathrm{~km}$ & con obstáculos \\
Urbano & $35 \mathrm{~m}$ & $9 \mathrm{~km}$ & línea vista \\
\hline
\end{tabular}

Tabla II. Pruebas de alcance.

2.4. Funcionalidad.- Se continúa el análisis con los experimentos realizados para probar el comportamiento del sistema para diferentes variables de interés del perímetro virtual. En estos experimentos, se seleccionaron cuatro puntos en el mapa formando un cuadrilátero convexo, y las coordenadas de estos puntos se configuraron como el perímetro virtual del NS. Luego, el NS se movió a lo largo del área del perímetro virtual, registrando los datos de relevancia (ubicación actual, fecha, hora y disparadores de estimulación).

En todas las pruebas realizadas se corroboró que los estímulos fueran activados de acuerdo con la ubicación del NS respecto al perímetro virtual, y los parámetros previamente configurados, verificando el correcto funcionamiento de todo el sistema.

2.5. Pruebas preliminares en ganado bovino.- Finalmente, se realizaron pruebas preliminares en bovinos (ver Fig. IX) usando diferentes versiones del dispositivo en cada etapa del desarrollo, probando varios aspectos de la solución propuesta. En estos experimentos, se colocó el collar durante algunas horas en bovinos, verificando la facilidad de instalación y que todos los componentes se mantuvieron en su lugar. 
También se realizó una validación funcional primaria observando las primeras reacciones de los animales. Se encontró que los materiales usados no producen ningún daño a los animales y no provocan molestias que afecten su comportamiento habitual.

Si bien el comportamiento observado varió con los distintos animales y las características de su entorno, se constató que gran parte de ellos no reaccionó al estímulo sonoro (buzzer). Sin embargo, se visualizó una reacción significativa al estímulo táctil (motor vibrador), en muchos casos causando su desplazamiento en el sentido deseado (hacia el interior del perímetro virtual) sin entrenamiento previo.

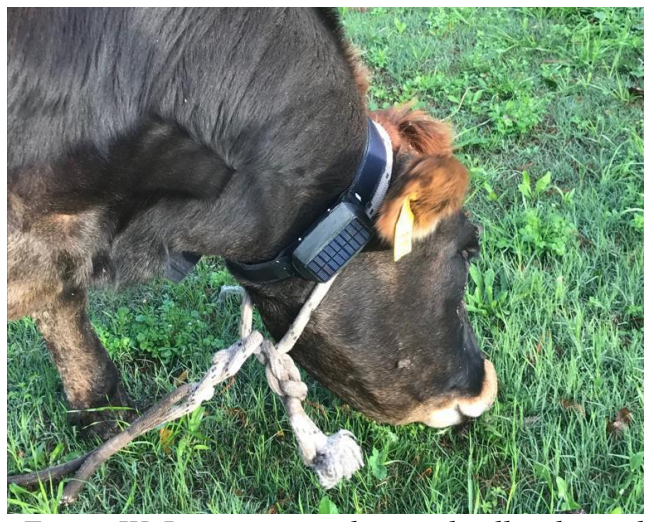

Figura IX. Bovino pastando con el collar diseñado.

2.6. Resumen de especificaciones.- En la Tabla III se presentan las principales características de la plataforma desarrollada. La precisión en la ubicación surge de la hoja de datos del GPS. El máximo período de muestreo se da cuando el NS se encuentra dentro de las franjas de estimulación, mientras que el típico surge del intervalo de tiempo que se seleccionó para transmitir (32 segundos), la adquisición del GPS y su procesamiento, y los tiempos correspondientes al protocolo de comunicación. El tiempo de respuesta representa el tiempo típico de demora entre que se cambia un parámetro de configuración en el servidor central, y toma efecto en el NS.

\begin{tabular}{ll}
\hline Especificación & Descripción \\
\hline Precisión (en ubicación) & $2.5 \mathrm{~m}$ \\
Período de muestreo & Mín: 1 segundo; Typ: 40 segundos \\
Alcance & $9 \mathrm{~km}$ \\
Protocolo de comunicación & LoRa - Clase A $(915 \mathrm{MHz})$ \\
Consumo de corriente & $28.1 \mathrm{~mA}$ \\
Autonomía & 5 días sin presencia de sol \\
& 10 días con $3 \mathrm{PSH}$ \\
& Sin limitaciones con $7 \mathrm{PSH}$ \\
Estímulos & Sonoro (buzzer) y táctil (vibrador) \\
Peso & $0.8 \mathrm{Kg}$ \\
Tamaño & Máx: 90 x 9.4 x $4.5 \mathrm{~cm}$ \\
Tiempo de respuesta & \\
(config. de parámetros) & Típico: 1 minuto \\
\hline
\end{tabular}

Tabla III. Resumen de especificaciones.

3. Discusión.- Si bien la autonomía del dispositivo es adecuada para una plataforma de investigación, podría resultar insuficiente para un producto comercial. En este aspecto, debe considerarse que tanto la estimación de consumo como la de energía cosechada que se realizó fueron conservadoras. Por otra parte, se espera que el dispositivo reduzca su consumo con el 
transcurso del tiempo, si se logra que el animal aprenda, mediante entrenamiento, a evitar los estímulos, permaneciendo más tiempo en la zona de menor consumo.

Puede verse en la Fig. X que el cuello de botella del consumo es el GPS. Por lo que, aumentar el tiempo que está apagado el NS (y en particular que lo está el GPS) en el modo de bajo consumo sería una mejora significativa. Por tanto, si fuese necesario aumentar la autonomía, debería considerarse aumentar la cantidad de paneles solares o utilizar un GPS que consuma menos.

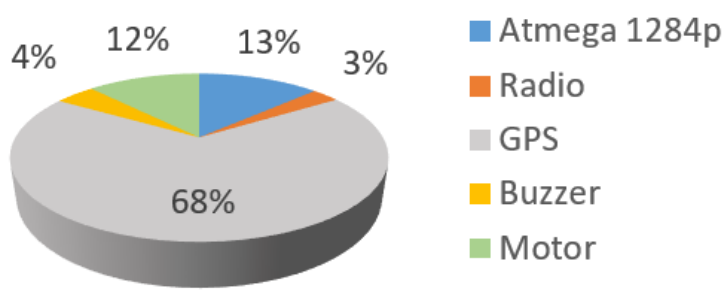

Figura X. Distribución del consumo del NS.

En cuanto al alcance, todavía falta realizar pruebas exhaustivas de la máxima distancia alcanzable de comunicación entre los NS y el gateway. Las pruebas realizadas en entornos rurales, llegaron a cubrir 1,6 km. Se cree que el resultado de esta prueba estuvo condicionado en gran medida por los obstáculos presentes, debido a que no se logró demasiada altura en la colocación del gateway. Sin embargo, se logró un alcance de $9 \mathrm{~km}$ en un entorno urbano con línea vista, por lo que se espera obtener resultados similares en un entorno rural, mejorando la instalación del gateway.

La plataforma permite visualizar de manera remota la ubicación de los NS y acceder al historial de sus últimos movimientos. Con este sistema, se pueden evaluar distintos esquemas de estimulación, determinando su desempeño en función de la cantidad de tiempo que se logra mantener confinado al animal en el perímetro establecido. Se destaca que se visualizó una reacción significativa al estímulo táctil. Se especula entonces con la hipótesis de que luego de un periodo de aprendizaje, los animales puedan moverse en una dirección predefinida, de modo tal que eviten el estímulo táctil, al escuchar primero el estímulo sonoro.

Respecto al confinamiento virtual de ganado, hay mucho trabajo por delante en materia de investigación interdisciplinaria. Este deberá incluir pruebas que validen la eficacia de las técnicas de confinamiento, teniendo en cuenta el bienestar animal y su productividad. En particular será necesario determinar el nivel de estrés que le generan al animal los estímulos, y el eventual impacto en la producción de carne o leche. Es una tendencia mundial la necesidad de utilizar estímulos que sean compatibles con el bienestar animal. Por esta razón, la plataforma propuesta cuenta con estímulos sonoros y táctiles, lo que la distingue de otras soluciones.

4. Conclusiones.- En este trabajo se presentó el diseño y fabricación de una plataforma para el confinamiento virtual de animales basado en estímulos sonoros y táctiles, lo que lo hace compatible con el bienestar animal.

El aporte principal de este trabajo fue crear una plataforma para la investigación de técnicas de confinamiento virtual de animales. Esta plataforma permite al investigador modificar varios parámetros de interés incluyendo el tamaño y ubicación del perímetro virtual, las zonas de estimulación, y los principales parámetros del generador de estímulos.

Las pruebas realizadas en animales, sugieren que el estímulo táctil no es inocuo, por lo que se cree, que la interacción del estímulo sonoro en conjunción con el táctil podría lograr el cometido de mantener confinado al animal, luego de un periodo de aprendizaje. La investigación sobre la eficacia de metodologías de confinamiento basadas en esta plataforma deberá ser realizada en una próxima etapa por un equipo interdisciplinario. 
Si se lograse encontrar un método efectivo de confinamiento virtual para ganado, son muchos los beneficios que podrán obtener los productores rurales. Entre ellos se destacan el pastoreo de precisión, la disminución tanto de la cantidad de alambrados internos como de la inversión en mantenimiento y la posibilidad de transportar el ganado sin intervención directa.

5. Agradecimientos. -Este proyecto fue apoyado por la Agencia Nacional de Innovación e Investigación (ANII), Fundación Julio Ricaldoni, el capítulo uruguayo de Circuitos y Sistemas IEEE, y PEDECIBA Informática. Los autores quisieran agradecer a TeLIoT por el soporte técnico brindado y préstamo de un gateway, a Facultad de Veterinaria (Udelar) y Dayanara Bonilla, por permitirnos el uso de sus instalaciones y ganado, y ofrecer asistencia en la realización del trabajo experimental. 


\section{Referencias}

[1] C. Umstatter, "Review: The evolution of virtual fences: A review," Comput. Electron. Agric., vol. 75 , no. 1, pp. 10-22, Jan. 2011.

[2] R. E. Marsh, "Fenceless animal control system using GPS location information," Feb. 9 1999, US Patent 5,868,100.

[3] A. Llaria, G. Terrasson, H. Arregui, and A. Hacala, "Geolocation and monitoring platform for extensive farming in mountain pastures," in IEEE Intl. Conf. on Industrial Technology (ICIT), Mar 2015, pp. 2420- 2425.

[4] S. J. Giunta, "Wireless fencing system," Dec. 28 2010, US Patent 7,856,947.

[5] Z. Butler, P. Corke, R. Peterson, and D. Rus, "Virtual fences for controlling cows," in IEEE Intl. Conf. on Robotics and Automation, vol. 2004, no. 5, 2004, pp. 4429-4436.

[6] _ , "From robots to animals: Virtual fences for controlling cattle," Intl. Journal of Robotics Research, vol. 25, no. 5-6, pp. 485-508, 2006.

[7] G. Bishop-Hurley, D. Swain, D. Anderson, P. Sikka, C. Crossman, and P. Corke, "Virtual fencing applications: Implementing and testing an automated cattle control system," Comput. Electron. Agric., vol. 56, no. 1, pp. 14-22, 2007.

[8] D. Campbell, J. Lea, W. Farrer, S. Haynes, and C. Lee, "Tech-savvy beef cattle? how heifers respond to moving virtual fence lines," Animals, vol. 7, no. 9, 2017.

[9] D. Campbell, J. Lea, S. Haynes, W. Farrer, C. Leigh-Lancaster, and C. Lee, "Virtual fencing of cattle using an automated collar in a feed attractant trial," App. Animal Behaviour Sc., vol. 200, pp. 71-77, 2018.

[10] I. Ferrer, "Holanda prohíbe el uso de collares electricos para perros (in spanish)," Diaro El País, 6/4/2019, disponible en línea, consultado 23/2/2021. https://elpais.com/elpais/2019/04/06/mundo_animal/1554560388_687751.html,

[11] N. Acosta, N. Barreto, P. Caitano, R. Marichal, M. Pedemonte, J. Oreggioni, "Research platform for cattle virtual fences", IEEE International Conference on Industrial Technology (ICIT), pags. 797-802, Buenos Aires, Argentina, 26-28 Feb, 2020.

[12] N. Acosta, N. Barreto, P. Caitano, "Plataforma de geolocalización e investigación en confinamiento virtual de bovinos", tesis de grado, IIE, Facultad de Ingeniería, Universidad de la República (Uruguay), 2019.

[13] Catel, "Cercas virtuales: hacia un futuro sin límites", disponible en línea, consultado 23/2/2021, http://catel.uy,

[14] IBM, LoRaWAN in C (LMiC) Technical Specification v 1.5, Mayo 2015.

[15] M. Kooijman, “Arduino-lmic library", 2017, disponible en línea, consultado 23/2/2021, https://github.com/matthijskooijman/arduino-lmic

[16] S. D. Roth, "Ray casting for modeling solids," Computer Graphics and Image Processing, Volume 18, Issue 2, Pages 109-144, 1982, 